\title{
Les soubresauts de l'Assemblée galloise : le caniche et le tigre
}

Troubled Times at the Welsh Assembly

\section{Monique Curcuru}

\section{OpenEdition}

\section{Journals}

Édition électronique

URL : http://journals.openedition.org/rfcb/700

DOI : $10.4000 /$ rfcb.700

ISSN : 2429-4373

Éditeur

CRECIB - Centre de recherche et d'études en civilisation britannique

\section{Édition imprimée}

Date de publication : 1 février 2002

Pagination : 44-50

ISBN : 2-911580-13-3

ISSN : 0248-9015

\section{Référence électronique}

Monique Curcuru, "Les soubresauts de l'Assemblée galloise : le caniche et le tigre », Revue Française de Civilisation Britannique [En ligne], XI-3 | 2002, mis en ligne le 21 mars 2016, consulté le 01 mai 2019. URL : http://journals.openedition.org/rfcb/700 ; DOI : 10.4000/rfcb.700

Ce document a été généré automatiquement le 1 mai 2019.

\section{(c) $(1)$}

Revue française de civilisation britannique est mis à disposition selon les termes de la licence Creative Commons Attribution - Pas d'Utilisation Commerciale - Pas de Modification 4.0 International. 


\title{
Les soubresauts de l'Assemblée galloise : le caniche et le tigre
}

\author{
Troubled Times at the Welsh Assembly
}

\section{Monique Curcuru}

1 Alors que le premier ministre britannique Tony Blair a fait bénéficier trois des composantes du Royaume-Uni d'une décentralisation relative, renouant ainsi avec la politique du gouvernement travailliste des années soixante-dix, il est intéressant de constater en France un renouveau du régionalisme celtique. La loi de décentralisation de 1982 avait entériné une Bretagne à quatre départements, séparée de la Loire-Atlantique. Un mouvement populaire se dessine actuellement en faveur de la réunification administrative de la Bretagne, mettant en avant la dynamique culturelle bretonne, qui débouche sur le développement économique. Les défenseurs du régionalisme breton estiment que l'identité est source de développement dans une économie mondialisée. Ils se fondent sur une majorité de $70 \%$ à $75 \%$ favorables au rattachement à cette nouvelle unité administrative. Cependant, le mouvement manque de dimension politique, bien que les fondateurs du Comité pour l'unité administrative de la Bretagne affirment que «le sentiment d'appartenance à un territoire est la condition de l'exercice de la démocratie »"

2 L'identité culturelle galloise est indéniable puisqu'un habitant sur cinq du pays de Galles parle la langue celtique. Cependant, la population est relativement instable, affectée par des flux migratoires et des transferts en direction, ou à partir, d'autres pays du RoyaumeUni. La décentralisation de certains pouvoirs législatifs visait dès les années soixante-dix à satisfaire les aspirations nationalistes de Plaid Cymru. On peut noter que les travaillistes, à l'origine du projet, étaient divisés sur la question, tandis que les conservateurs y étaient hostiles.

3 L'échec du projet lors du référendum de 1979 était dû notamment aux moindres compétences attribuées à l'Assemblée galloise; la position de Plaid Cymru était très réservée sur certains aspects du texte de loi. Cependant, une majorité de la population est devenue favorable dans les années quatre-vingt et quatre-vingt-dix à la constitution d'une Assemblée galloise démocratiquement élue. Il faut ici tenir compte de l'influence du 
ministère aux Affaires galloises (Welsh Office) et parallèlement de la diminution du prestige et des responsabilités du pouvoir local, c'est-à-dire des conseils de district et de comté.

4 Les conservateurs ont introduit en 1995 de nouvelles unités administratives, considérées comme plus efficaces, moins coûteuses à gérer et plus proches des habitants. Mais comme les quatre ministres aux Affaires galloises qui se sont succédé durant le gouvernement conservateur n'étaient pas des députés gallois, le parti nationaliste Plaid Cymru considère que le pays de Galles était administré par un " gouverneur général », qui ne tenait aucun compte des souhaits de la population. De fait, aucun député conservateur n'a été élu au pays de Galles aux élections générales de 1997, ni en 2001.

5 Après la victoire des travaillistes, la décentralisation a été conçue différemment pour l'Écosse et le pays de Galles: l'Assemblée galloise devait être limitée à un rôle de discussion et de consultation, tout en disposant d'un pouvoir de contrôle sur le budget dévolu au ministère des Affaires galloises. Ron Davies, alors ministre et dirigeant du parti travailliste, considérait la décentralisation comme « un processus » et non comme « un événement ", mais selon Tom Nairn, le gouvernement travailliste a une conception totalement opposée ${ }^{2}$.

Le premier obstacle rencontré par Tony Blair sur la voie de la décentralisation au pays de Galles a été la succession de Ron Davies après sa démission pour affaires de mœurs en octobre 1998. Le premier ministre a nommé Alun Michael, député de la circonscription de Cardiff South et Penarth, au poste de ministre ; le parti travailliste gallois s'est divisé sur sa candidature, la base soutenant la candidature du député de la circonscription de Cardiff Ouest, Rhodri Morgan, dissident. Alun Michael n'a été élu que grâce aux votes secrets des syndicats.

7 William Rees Mogg a souligné dans le Times ${ }^{3}$ que Blair avait lui-même été élu dirigeant du parti travailliste selon le principe d'une voix par syndiqué : le retour au principe du vote bloqué a, au contraire, permis à trois dirigeants syndicaux de faire élire Alun Michael. Pour la majorité des travaillistes gallois, favorables à Rhodri Morgan, la décentralisation pouvait apparaitre comme une supercherie, alors que la suprématie anglaise était maintenue.

\section{L'Assemblée : pouvoirs et composition}

8 Selon la loi de 1998 (Government of Wales Act), l'Assemblée galloise, organisme législatif responsable, élue pour quatre ans, ne dispose que de pouvoirs législatifs secondaires, c'est-à-dire du droit de modifier les lois concernant le pays de Galles votées par le Parlement britannique. Elle acquiert les pouvoirs économiques et sociaux précédemment exercés par le ministre aux Affaires galloises, c'est-à-dire le développement économique, l'agriculture, l'industrie et la formation, l'enseignement, l'administration locale, la santé, les services sociaux, le logement, l'environnement, les transports, ainsi que la langue galloise. Le gouvernement britannique conserve ses pouvoirs en matière de politique intérieure (immigration, sécurité sociale), de politique économique et de politique étrangère.

Bien que l'Assemblée n'ait pas de pouvoirs fiscaux, et dépende du Trésor britannique sur le plan financier, elle dispose de pouvoirs législatifs secondaires étendus, et c'est la première fois depuis l'union avec l'Angleterre que le pays de Galles en tant que nation 
exerce un contrôle démocratique sur certains domaines de la vie publique. C'est un organisme hybride, au sein duquel l'exécutif de huit membres, composé sur le modèle du cabinet britannique, est responsable devant un certain nombre de commissions. Celles-ci contribuent à la représentation démocratique symbolisée par l'Assemblée elle-même, puisque les deux tiers des présidents de commissions appartiennent à des partis d'opposition.

En outre, des représentants d'organisations extérieures et des fonctionnaires sont conviés aux débats des commissions. L'Assemblée en effet travaille en triple partenariat avec l'administration locale, les entreprises et le secteur caritatif. Un quatrième partenariat unit l'Assemblée au ministre des Affaires galloises qui représente le cabinet britannique et qui est convié par l'Assemblée à débattre du programme législatif du gouvernement concernant le pays de Galles.

11 L'Assemblée est également intégrée au processus de décentralisation réalisé dans les autres composantes du Royaume-Uni par l'intermédiaire de concordats signés avec le gouvernement britannique et l'exécutif écossais. Enfin, au niveau européen, elle travaille en liaison avec le ministère des Affaires étrangères et ses représentants font également partie de la délégation de négociateurs britanniques; en outre, l'Assemblée dispose d'un bureau à Bruxelles.

Le pays de Galles est évidemment très attaché à la question des langues minoritaires et plus généralement à celle du régionalisme culturel, civique et économique. Des missions commerciales ont été organisées en Europe et des liens sont établis avec les quatre régions motrices d'Europe. La politique de régénération économique et sociale mise en œuvre par l'Assemblée dépend largement des fonds de « l'objectif Un ». Le pays de Galles voudrait, grâce à l'aide européenne, devenir un deuxième "tigre celte » après la République d'Irlande.

13 L'Assemblée est une chambre parlementaire de soixante membres élue au suffrage universel direct selon le système uninominal à un tour pour quarante sièges, les vingt autres étant pourvus au scrutin proportionnel à partir de listes régionales. Il s'agit d'un mode de scrutin révolutionnaire en Grande-Bretagne puisque les précédentes élections, au niveau local, national ou européen, s'étaient déroulées au scrutin majoritaire à un tour. Lors de la campagne préélectorale au printemps 1999, les dirigeants travaillistes ne purent que constater l'augmentation du soutien pour les nationalistes gallois. Les débats principaux ont donc porté sur le programme de Plaid Cymru : alors que les travaillistes le présentaient comme un parti indépendantiste, Dafydd Wigley le situait au centre gauche, donc à la même place sur l'échiquier politique que les travaillistes, et avançait le principe d'autonomie pour le pays de Galles et son intégration au sein d'une Europe unie.

14 Tandis que les travaillistes et le Plaid Cymruu restaient très proches dans leurs programmes, le dirigeant gallois conservateur Rod Richards représentait la tendance de la droite eurosceptique. L'impopularité relative du dirigeant travailliste Alun Michael au pays de Galles, considéré comme le représentant du gouvernement britannique, tout autant que la popularité des nationalistes, explique que les travaillistes avec vingt-huit sièges n'aient pas obtenu la majorité absolue, tandis que Plaid Cymru constituait numériquement le deuxième groupe à l'Assemblée avec dix-sept sièges, neuf sièges seulement revenant eux conservateurs et six aux libéraux-démocrates.

15 Le parti travailliste est minoritaire dans chaque commission. Ses résultats électoraux s'expliquent d'abord par le manque d'intérêt de l'électorat, puisque l'Assemblée n'avait 
initialement obtenu le soutien que du quart de l'électorat et de la moitié des votants. Les conservateurs ont réalisé leur meilleur score dans le Sud-Est du pays de Galles, partie la plus rurale et la plus anglicisée qui avait le plus souffert du déclin de l'agriculture. Les voix travaillistes ont diminué de moitié dans chaque circonscription par rapport aux élections de 1997. L'Independent voyait ainsi dans l'élection " la plus grande défaite électorale subie par Tony Blair depuis son arrivée au pouvoir $»^{4}$, tandis que Dafydd Wigley se félicitait du "tremblement de terre politique qui prenait de l'ampleur depuis plusieurs semaines" ${ }^{5}$. Le monopole travailliste dans le sud urbain a effectivement été détruit par Plaid Cymru.

\section{Les travaillistes}

$16 \mathrm{Au}$ lendemain des élections, les travaillistes ont proposé un triple partenariat aux nationalistes et aux libéraux-démocrates, leur offrant des postes à responsabilité. Les libéraux-démocrates ont mis comme condition, entre autres, la nécessité de réduire les délais pour les consultations hospitalières, ainsi que le nombre d'élèves pour les classes dans l'enseignement primaire, ce qui correspondait à la politique travailliste. Néanmoins, Alun Michael opta finalement pour un gouvernement minoritaire, tenant compte du petit nombre de sièges des libéraux-démocrates, et qui permettait au parti travailliste de se distinguer des deux partis de l'opposition dont le programme est le plus proche du sien. Les libéraux-démocrates se sont vu ainsi privés, au pays de Galles comme dans le Royaume-Uni, d'une possibilité de participer au gouvernement.

17 La faiblesse de la position travailliste a été aggravée au début de l'année 2000 : les partis d'opposition critiquaient le régime autoritaire imposé par Alun Michael, premier secrétaire, et lui reprochaient surtout la situation financière du pays de Galles, dont la subvention européenne pour les six ans à venir n'était pas garantie. Le premier ministre britannique était bien parvenu à obtenir des fonds «Objectif Un» pour les régions défavorisées du pays, mais ceux-ci doivent être contrebalancés par une aide accordée par le Chancelier de l'Échiquier.

La crise politique qui s'est ouverte à l'Assemblée galloise lorsqu'Alun Michael a démissionné au début de février 2000 sous la menace d'une motion de censure a eu des répercussions en Grande-Bretagne. Les conservateurs ont essayé d'en tirer avantage, mais le premier ministre les a accusés de chercher à perturber le fonctionnement de l'Assemblée en faisant cause commune avec les nationalistes: «la décision appartient aux Gallois, mais les gens doivent se demander pourquoi nationalistes et Tories se retrouvent dans le même bain ». Alun Michael estimait "qu'il revenait au parti travailliste de décider qui devait être présenté à l'Assemblée pour occuper les fonctions de premier secrétaire $\|^{6}$.

19 Le successeur évident d'Alun Michael était Rhodri Morgan, initialement écarté par Blair en raison de ses positions non orthodoxes mais soutenu par la majorité du parti travailliste gallois et des députés travaillistes à l'Assemblée. Un groupe de dissidents travaillistes a même fait connaître son opposition à une nouvelle nomination d'Alun Michael. Un autre élément qui plaidait en faveur de Morgan aux yeux des dirigeants travaillistes était le soutien qu'il avait apporté à son prédécesseur qui lui avait confié la responsabilité du développement économique et des relations avec l'Europe.

20 Le nouveau premier secrétaire s'est d'abord efforcé de rétablir le calme, demandant que "cesse l'abattage des premiers secrétaires »" Il a, à son tour, envisagé un gouvernement de coalition, cette fois limité aux libéraux-démocrates, sur le modèle du gouvernement 
écossais. L'accord a effectivement été signé à l'automne 2000, les libéraux-démocrates bénéficiant de deux postes dans l'exécutif dont celui de premier secrétaire adjoint.

Morgan espérait ainsi apporter une plus grande stabilité à l'administration galloise, tandis que les libéraux-démocrates comptaient faire accepter l'essentiel de leur programme pour le pays de Galles, notamment dans les domaines de l'enseignement et de la santé. Le changement de premier secrétaire qui a dû être entériné par le gouvernement britannique puisqu'il s'est fait suivant le processus démocratique a, de toute évidence, modifié la scène politique galloise.

\section{Les nationalistes}

Dès l'entrée en fonction de l'Assemblée, le parti du pays de Galles ou Plaid Cymru insistait sur la nécessité d'un consensus à la fois au sein de celle-ci et de la population. L'union des partis d'opposition contre le premier secrétaire n'existe plus depuis l'élection de Rhodri Morgan, dont Dafydd Wigley reconnaissait qu'il n'avait rien «d'un caniche de Downing Street $»^{8}$. En outre, le succès de Plaid Cymru aux élections à l'Assemblée galloise ne se retrouve pas au niveau des élections britanniques.

En juin 2000, Dafydd Wigley, après avoir démissionné pour raison de santé, a été remplacé par Ieuan Wyn Jones ; le nouveau dirigeant voulait étendre l'électorat nationaliste au-delà des régions du nord et de l'ouest où sont concentrés les habitants qui parlent gallois.

La poursuite de la politique de consensus avec les travaillistes à l'Assemblée n'a pas empêché ces derniers d'accuser la direction du parti nationaliste de renier sa tradition socialiste radicale pour revenir à un programme rural conservateur.

Les nationalistes constituent non seulement une menace directe pour les travaillistes au pays de Galles; ils souhaitent de plus obtenir la transformation de l'Assemblée en Parlement disposant des mêmes pouvoirs législatifs que le Parlement écossais, notamment celui de lever l'impôt, revendication soutenue par les libéraux-démocrates. Bien que non indépendantiste, Plaid Cymru, s'inspirant du modèle catalan, propose la création au Parlement européen d'une deuxième chambre qui représenterait les petites nations.

\section{L'avenir de l'Assemblée galloise}

En juin 1999, Dafydd Wigley comparait, non sans fierté, l'Assemblée nationale de Paris et celle de Cardiff, bien que cette dernière ne soit "ni aussi grande ni aussi impressionnante ". De fait, Rhodri Morgan déclarait aussi en octobre 2000 que l'Assemblée galloise était " une nouvelle et toute jeune institution ${ }^{10}$. Cependant, elle symbolise l'identité nationale galloise et constitue donc un enjeu pour les quatre partis qui y sont représentés, y compris les conservateurs qui étaient initialement opposés à la décentralisation.

Comme l'écrit Tom Nairn, elle doit, en fait, construire, ou reconstruire, une nation, mais le but que se fixent les nationalistes, à savoir la parité avec le Parlement écossais, confrontera le pays de Galles au nouveau problème de ses rapports avec le Parlement de Westminster. Bien qu'elles tiennent une place importante dans le manifeste électoral travailliste de 1997, les réformes constitutionnelles, qui se sont heurtées à de nombreux 
obstacles dans les différentes composantes du Royaume, ne semblaient pas être défendues avec toute la conviction nécessaire par le premier ministre.

Les restrictions apportées à la décentralisation rendent difficile la politique de consensus au sein de l'Assemblée galloise : durant l'été 2000, l'ancien ministre des Affaires galloises, Ron Davies, a estimé que l'Assemblée avait à ses débuts la capacité de "prendre part aux grands matchs " et se contentait aujourd'hui de commenter les événements sur la touche, les partis politiques d'opposition prenant des décisions que l'Assemblée n'avait pas le pouvoir de mettre en pratique ${ }^{11}$. Pour Ron Davies, il importe de remettre en question le gouvernement gallois dans la perspective des élections générales de 2005.

Comme le Parlement écossais, l'Assemblée galloise a adopté une politique progressiste en matière d'égalité des chances. Jouant pleinement la carte des nouvelles technologies de communication, elle travaille au renforcement de ses relations avec la Hongrie, l'Italie et la Catalogne, dans une optique commune de diversité culturelle.

Avec la dévolution, le Royaume-Uni a cessé d'être un État unitaire monolithique, et s'est engagé sur la voie d'une représentation plus démocratique. Le statut actuel de l'Assemblée galloise est certes mal déterminé puisqu'elle se situe entre les Conseils généraux français et les assemblées des Länder allemands. En dehors des considérations politiques, on peut espérer, avec Dafydd Wigley, qu'elle parviendra «à réaliser une qualité et un niveau de vie qui encouragent les jeunes à rester au pays ».

\section{NOTES}

1. Le Monde, 3 juillet 2001.

2. Tom NAIRN, After Britain, New Labour and the Return of Scotland, Londres : Granta Books, 2000.

3. Cité par Tom NAIRN, ibid.

4. 8 mai 1999

5. Ibid.

6. The Independent News UK, 9 février 2000.

7. The Independent, 16 février 2000.

8. Ibid, 10 février 2000.

9. Intervention lors $\mathrm{du}$ colloque "Devolution and the election of the Scottish Parliament and the National Assembly for Wales", British Council, Paris, 2 juin 1999.

10. The Independent, 6 octobre 2000.

11. Intervention lors $\mathrm{du}$ colloque "Devolution and the election of the Scottish Parliament and the National Assembly for Wales”, British Council, Paris, 2 juin 1999. 


\section{RÉSUMÉS}

Les dirigeants britanniques ont, dès les années soixante-dix, amorcé un mouvement de dévolution qui ne faisait pas l'unanimité. Malgré l'échec du référendum de 1979, le mouvement s'est poursuivi jusqu'à la mise en place de l'Assemblée galloise en 1999. Les élections galloises n'ont cependant pas réglé tous les problèmes. Manquant de peu la majorité absolue, les travaillistes ont dû composer avec les formations d'opposition. En outre les crises qui ont secoué la tête de l'exécutif ont pesé sur la gestion de l'espace autonome réduit dévolu au pays de Galles, lequel réclame aujourd'hui un statut identique à celui de l'Écosse, tout en espérant d'une insertion accrue dans l'Union européenne ce surplus d'indépendance que Londres n'est pas prêt à lui accorder dans l'immédiat.

By the 1970s British political leaders had initiated a movement towards devolution, which was by no means an object of consensus. Despite the failure of the 1979 referendum, demands for devolution culminated in the setting up of the Welsh Assembly after the 1997 General Election. But the Welsh elections of May 1999 did not solve all the problems. Three seats short of an absolute majority, the Welsh Labour Party has had to make deals with the opposition parties. Moreover, the successive crises which engulfed the first leaders of the Welsh Executive have weighed heavily upon the management of the limited political autonomy devolved to Wales. The Welsh are now aiming to get a status similar to that of Scotland, while pinning their hopes for increased autonomy - which Downing Street is not ready to grant - on further integration within the European Union.

\section{AUTEUR}

\section{MONIQUE CURCURU}

Université Stendhal - Grenoble III 